\title{
EDITORIAL
}

\section{Redaction, retraction and reaction}

\author{
In our final Editorial of the year, we take a look back at 2012 and two of the main microbiology \\ stories that made the news.
}

A glance at some of the main microbiology-related headlines for the year might suggest that 2012 was something of an annus horribilis for microbiology, but a detailed look at the stories behind these headlines paints a more subtle picture of the scientific process at work.

At the start of the year, the biggest story was the intervention of a US government advisory board, the National Science Advisory Board for Biosecurity (NSABB), in the publication of two influenza papers. The papers, one submitted to Nature and one to Science, reported that mutated versions of highly pathogenic avian influenza (HPAI) H5N1 virus were transmissible between ferrets via the respiratory route. Although both journals had carried out their own dual-use biosecurity assessments on the work, the papers came to the attention of the NSABB, and the board took the unprecedented step of recommending they be redacted. It quickly became clear, however, that there was no mechanism by which redacted papers could be published while still allowing legitimate scientists to access the redacted data. In March 2012, following much discussion and debate in many different forums, including meetings organized by the WHO and the Royal Society, the board reconvened to consider revised versions of the papers and this time voted for publication in full, although the decision was not unanimous. The papers were subsequently published $^{1,2}$.

This episode raised many issues which have yet to be resolved. In January 2012, influenza scientists declared a 60-day voluntary moratorium on research into HPAI transmission, allowing the researchers engaged in this work to fully explain the measures that had been taken to protect themselves and the public at large, as well as the benefits of the work for public health. These elements were both discussed extensively in the published papers, but the moratorium was subsequently extended indefinitely to allow institutions, funding bodies and governments worldwide to assess what biosafety and biosecurity requirements they deem necessary for such research to continue. Much of this work has been completed, and the continued moratorium was discussed in a recent series of commentaries in $\mathrm{mBio}$, with the authors of the original papers calling for it to be lifted for those researchers who have obtained full governmental and instituitional approval to continue working ${ }^{3}$.
The furore over the redaction also highlighted a key area of concern in science in general: the need for clear, consistent communication with key stakeholders and the public. Last month saw the culmination of a 2-year US National Institutes of Health-funded project to investigate the association between two viruses, xenotropic murine leukaemia virus-related virus (XMRV) and polytropic murine leukaemia virus (pMLV), and chronic fatigue syndrome $(\mathrm{CFS})^{4}$. The study, coordinated by Ian Lipkin of Columbia University, New York, USA, was instigated after the publication of a suite of studies failing to replicate the findings of the original papers ${ }^{5,6}$. Many observers questioned why this study was not discontinued when it became clear that XMRV was a recombinant that had been created accidentally by earlier laboratory experiments and that contamination with mouse DNA was common in PCR reagents, and both of the original papers had been retracted. However, many of the patients with CFS were still convinced of the link, so it was important, if expensive, to settle the issue once and for all, without any lingering doubt. In deference to the need for a clear-cut result, Lipkin took extreme care with the study design to ensure that the association was fairly tested. Every element of the multicentre, blinded study, from the diagnostic criteria used to select patients for inclusion, to the criteria used to call a sample positive or negative, was agreed by all the authors in advance, and crucially, all of the principal investigators involved in the original work participated. Each laboratory involved was free to use its own protocols for the molecular or serological detection of the viruses. The study involved 147 patients with CFS and 146 case-matched controls, and found no evidence for an association between either virus and CFS. This was of course an expensive exercise, but these conclusive results were endorsed by all the authors and cannot be misinterpreted. In the area of 'pathogen dediscovery', there are many examples of lingering doubts and missed opportunities that have created untold damage, and in this case, although the horse had bolted, it was right to close the barn door firmly behind it.

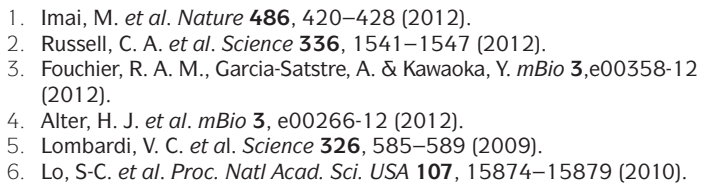

